\title{
Las pinturas egipcias de Malkata: ¿arte egipcio con sabor minoico? Una nueva perspectiva sobre las pinturas del palacio de Amenofis III y las influencias del Egeo
}

\author{
Inmaculada VIVAS SAINZ \\ Universidad de Educación a Distancia, UNED \\ Departamento de Historia del Arte, Facultad de Geografía e Historia, Madrid \\ ivivas@geo.uned.es; inmaculadavivas@hotmail.com
}

\begin{abstract}
RESUMEN
Nuestro estudio analiza las pinturas halladas en el palacio de Amenofis III en Malkata, y en particular su iconografía, entre la que encontramos temas tradicionales de la pintura mural egipcia junto con otros que reflejan la influencia del arte del Egeo, como motivos geométricos y con espirales, animales en la postura de galope tendido o símbolos como la cabeza de toro con una roseta. Rastrearemos el origen de esos nuevos elementos, buscando paralelos en el arte egipcio, egeo y próximo-oriental y analizaremos los medios de transmisión. Las pinturas palaciales de Tell el Dab'a (Avaris) de inicios de la XVIII dinastía, con técnica y estilo del Egeo, serán una importante conexión y una posible fuente de influencia directa sobre las pinturas de Malkata.
\end{abstract}

Palabras clave: Pinturas; egipcias; Malkata; influencia; egea.

\section{The Malkata paintings: Egyptian Art with a Minoan flavour? A new perspective on the paintings of the palace of Amenhotep III and its Aegean connections}

\begin{abstract}
Our study will focus on the paintings found on the palace of Amenophis III at the site of Malkata, analyzing their iconography which includes traditional Egyptian themes and also new elements which reflect the influence of Aegean Art such as geometric and spirals decoration, animals rendered in "flying gallop" and symbols like the bull head with a rosette between its horns. We will trace the origin of these symbols, looking for similar examples on the iconography of Egypt, the Aegean and the Near East, analyzing the means of transmission as well. The paintings unearthed at Tell el Dab'a (Avaris) in a palace of the early XVIII Dynasty, with remarkable Aegean style and technique, will prove to be an important connection and a possible direct influence for the Malkata paintings.
\end{abstract}

Key words: Egyptian; Paintings; Malkata; Aegean; Influence. 
Hace exactamente 100 años, durante los primeros meses de 1911, un equipo del $\mathrm{Me}$ tropolitan Museum of New York excavaba en Tebas los restos del palacio construido por Amenofis III, donde se hallaron cientos de fragmentos de pinturas murales. En los años posteriores el trabajo de la misión del Metropolitan Museum se centró en la excavación de distintas zonas del palacio, sacando a la luz numerosos restos de las pinturas que decoraban profusamente las salas del palacio, algunas de ellas incluso con decoración en las paredes, techos y suelos. Curiosamente en la memoria de excavación de 1915 Hugh Evelyn White escribía: "en uno de los dormitorios reales el diseño del techo estaba compuesto de espirales que rodean el símbolo de la cabeza de vaca con una roseta entre los cuernos, un símbolo conocido en el arte egeo de este período". La vinculación entre la iconografía de las pinturas de Malkata y el Egeo que entonces se señalaba, parece ser hoy mucho más sólida, como intentaremos demostrar.

En este breve estudio analizaremos una serie de elementos iconográficos peculiares que aparecen en las pinturas murales de Malkata del palacio fechado en el reinado Amenofis III (1391-1353 a.C.). Las ruinas del palacio se redescubrieron en 1888 por el egiptólogo francés Daressy. Más tarde, como ya hemos mencionado, las excavaciones en la zona fueron promovidas por el Metropolitan Museum of Art $(1910-1920)^{1}$ y por el University Museum of Pennsylvania en los años $70^{2}$. Desde la década de los 70 la misión japonesa de la Universidad de Waseda lleva a cabo las excavaciones en Malkata, habiendo desarrollado un ambicioso proyecto de estudio y divulgación ${ }^{3}$. En la década de 1980 durante el transcurso de las excavaciones de la misión de la Universidad de Waseda, se inició el análisis exhaustivo de los restos de pinturas del palacio de Malkata, en estado muy fragmentario.

Los restos arqueológicos arquitectónicos encontrados en Malkata son también muy fragmentarios, aunque se distingue un gran complejo palacial, en el que destacan el palacio del norte, el del sur, el palacio del rey y el palacio central distribuidos en torno a un gran patio. El conjunto palacial de Malkata incluye no sólo espacios representativos o públicos, sino también numerosas salas residenciales, y otras zonas auxiliares como almacenes, despensas, etc. ${ }^{4}$ Los numerosos fragmentos de pinturas

1 WINLOCK, Herbert E., "The Work of the Egyptian Expedition", en Bulletin of the Metropolitan Museum of Art, New York, n7, 1912, pp. 185-189; EVELYN-WHITE, Hugh G., "The Egyptian Expedition", en Bulletin of the Metropolitan Museum of Art, New York, nº10, 1925, pp. 253-256.

2 KAWAMURA, Kiichi A., "Preliminary Report of Excavations by the Waseda University Expedition Party at Malkata, Luxor, Egypt: The Second Season, Dec. 1972 - Jan. 1973”, en Orient, n¹2, Tokyo, 1976, pp. 15-26; WATANABE, Yasutada y SEKI, Kazuaki, The Architecture of "Kom El Samak" at Malkata-South: A Study of Architectural Restoration, Studies in Egyptian Culture, 5, Waseda University, Tokyo, 1986.

3 KEMP, Barry, "A Building of Amenophis III at Kôm El-'Abd", en Journal of Egyptian Archaeology, 63, 1977, pp. 71-82; KEMP, Barry y O’CONNOR, David, "An Ancient Nile Harbour: University Museum Excavations at the Birket Habu", en International Journal of Nautical Archaeology and Underwater Exploration, nº3, 1974, pp. 101-136; KEMP, Barry, "University Museum, University of Pennsylvania, Excavations at Malkata", en Newsletter of the American Research Center in Egypt, 1974, pp. 13-18.

4 Sobre la funcionalidad de las distintas zonas, véase el reciente estudio KOLTSIDA, Aikaterini, "Malkata Revisited: Defining Domestic Space at the Palace City of Amenhotep III", en GOYON, Jean-Claude y CARDIN, Christine (eds.), Proceedings of the Ninth International Congress of Egyptologists, Grenoble , 6-12 September 2004, vol. I, Leuven, Peeters Publishers, 2007, pp. 1011-1022. 
murales hallados in situ ayudaron además a identificar las distintas funciones de las salas del palacio, lo que indica que la temática decorativa está ligada al uso de la sala y que la elección de motivos no es casual, algo habitual en el arte egipcio.

El reinado de Amenofis III se caracterizó por el florecimiento de una próspera etapa de la historia egipcia, ya que durante varias décadas Egipto vivió en paz, disfrutando de una sólida posición en el ámbito político y desarrollando un destacado comercio exterior. En el terreno artístico hubo además una importante actividad. Amenofis III fue el primer rey de la XVIII dinastía que de manera destacada reflejó la posición dominante de Egipto mediante la construcción de gigantescos edificios conmemorativos y la promoción de un arte refinado. En este arte refinado parecen intuirse algunas influencias del exterior, lo que es lógico teniendo en cuenta el activo contacto entre Egipto y los otros grandes poderes del Próximo Oriente y del Mediterráneo oriental durante el reinado de Amenofis III 5 .

Para poder hacernos una idea de cómo debió de ser el complejo palacial cuando se construyó, debemos analizar con detalle sus restos. Los edificios palaciales de Malkata estaban construidos en adobe y revocados, para luego ser decorados con llamativas y variadas pinturas murales. Nos llama la atención que no sólo estaban decoradas las zonas públicas de los palacios, tales como las salas de audiencias con una clara función representativa de la realeza, sino también las zonas privadas, incluso las de menor importancia. En nuestra opinión, ello podría ser indicativo de que en Malkata existía una verdadera escuela de artistas, encargados exclusivamente de decorar las distintas zonas del palacio, hasta las de importancia secundaria.

Pero centrémonos en la variada temática iconográfica de las pinturas. Por ejemplo, en la sala de audiencias descubierta por Daressy existía un pavimento pintado con una escena de una zona pantanosa con plantas de papiro, con representaciones de patos, peces y aves. El techo se decoraba con rosetas azules y rojas y con llamativas espirales amarillas. Esas escenas nilóticas, con papiros y aves locales son muy características de la pintura egipcia. Los escalones del trono real estaban decorados con las representaciones de arcos y de cautivos, un motivo iconográfico egipcio también muy habitual, que simboliza el poder real y la dominación de los enemigos ${ }^{6}$.

En la gran sala central del palacio se hallaron 16 bases de columnas de caliza que debían soportar dos filas de columnas de madera. En el muro sur de la sala había una representación del rey en su trono, sin duda reflejo de las escenas habituales que debían de decorar la sala del trono. Lamentablemente no se han conservado restos de la decoración de esta sala, que sin duda debía de seguir ese mismo programa iconográfico de exaltación del poder real del monarca Amenofis III.

Cabe destacar la decoración de las otras zonas del palacio, cuya funcionalidad estaba más ligada al ámbito privado, donde se introducen nuevos temas. Como por

5 CLINE, Eric, "Amenhotep II and the Aegean: A Reassesment of Egypto-Aegean Relations in the $14^{\text {th }}$ Century", en Orientalia, n $^{\circ}$ 56, 1987, pp. 1-35; CLINE, Eric, "Contact, Trade or colonization? Egypt and the Aegean in the $14^{\text {th }}$ and $13^{\text {th }}$ Centuries B.C.", en Minos, $n^{\circ} 25-26,1990-91$, pp. 7-36; KOZLOFF, Arielle P. (ed.), Aménophis III. Le pharaon-soleil, París, Réunion des Musées Nationaux, 1993.

6 Sobre las representaciones de cautivos en el arte egipcio, véase SLIWA, Joachim, "Some Remarks concerning Victorious Ruler Representations in Egyptian Art", en Forschungen und Berichte. Archäologische Beiträge, n¹6, 1974, pp. 97-117. 
ejemplo el dormitorio real, donde hay una decoración en el techo con cabezas de toro, rosetas y espirales, en una composición geométrica que quizás tiene un aire más masculino y menos naturalista en contraposición a otras temáticas decorativas. Destaca sobre todo este motivo en concreto de rosetas entre cabezas de toro (fig. 1), motivo que apenas se atestigua previamente en el arte egipcio (aunque sí hay representaciones similares posteriores en tumbas ramésidas de Deir El- Medina). Las rosetas no eran un elemento decorativo muy utilizado tradicionalmente por los artistas egipcios, pero sí tienen buenos paralelos en los frescos del Egeo. Gracias a los restos de frescos del Egeo sabemos que las rosetas eran un elemento muy común en la decoración para formar composiciones, como por ejemplo lo vemos en los conocidos frescos de Akrotiri (fig. 2). Como motivo iconográfico, las rosetas se atestiguan en el Egeo también en las artes menores, lo que refuerza la idea de que este motivo no procede del repertorio egipcio. Curiosamente se halló un motivo de rosetas en la decoración de la tumba de Hepzefa en Assiut datada en el Reino Medio, que se ha interpretado por varios autores como una decoración con influencia del arte textil del Egeo?

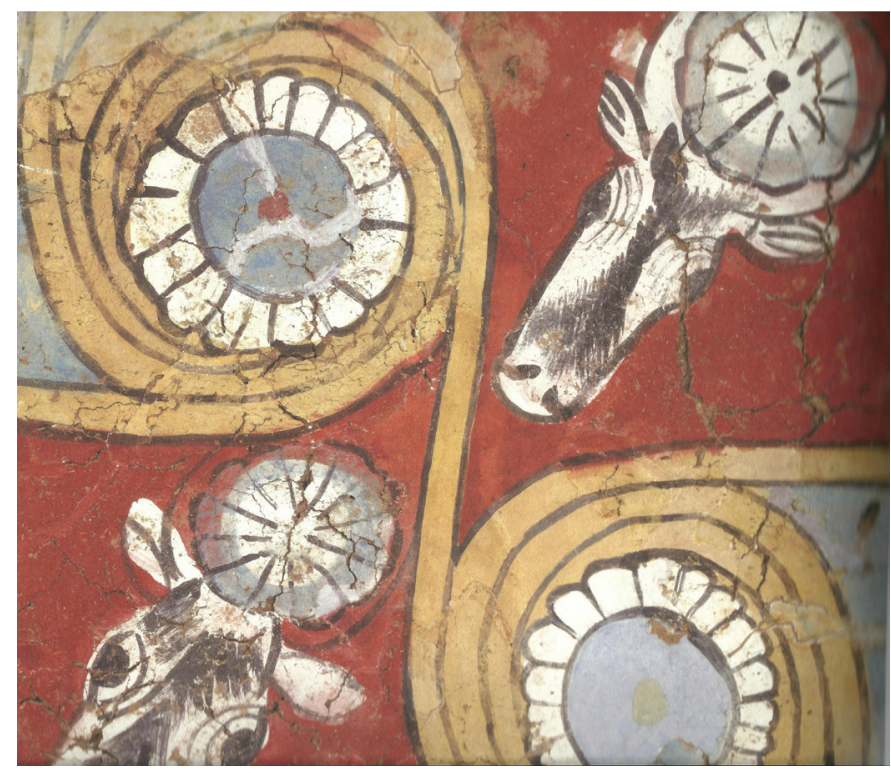

Fig. 1. Decoración del techo del dormitorio real del palacio de Malkata, Reinado de Amenofis III, Nueva York, Metropolitan Museum of Art.

En el techo del dormitorio real había un motivo de un buitre con las alas desplegadas, junto con el nombre de coronación de Amenofis III, motivo muy recurrente en la iconografía egipcia, posiblemente reflejando protección sobre el monarca (una

7 SHAW, Maria, "Ceiling Patterns from the tomb of Hepzefa", en American Journal of Archaeology, 74, 1970, pp. 25-30. 


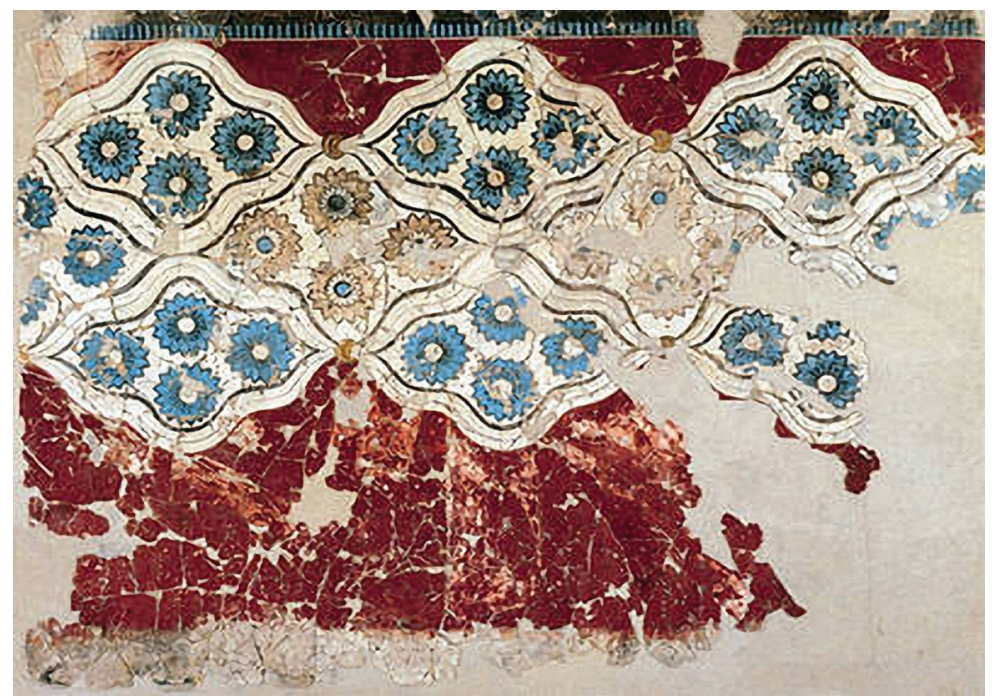

Fig. 2. Fresco de Akrotiri con decoración de rosetas, ca. 1500 A.C., Atenas, Museo Arqueológico Nacional.

temática muy apropiada tratándose del dormitorio del rey). Este motivo se documenta también en la decoración de los techos de templos del Reino Nuevo ${ }^{8}$. Nos llama la atención la habilidad del artista para adaptar el motivo al tipo de contexto, un techo, mostrando las figuras de los buitres con las alas extendidas, en una postura majestuosa pero que sobre todo está pensada para ser contemplada desde abajo, como si los buitres sobrevolasen el cielo9. En este contexto iconográfico, el buitre con alas extendidas es también probablemente símbolo del jeroglífico egipcio que significa "cielo". En este caso, el motivo iconográfico funciona probablemente como símbolo de protección, vinculándose además la figura del buitre con la diosa Nekhbet, símbolo del Alto Egipto. Cabe señalar también que en numerosas partes del palacio encontramos en la decoración el nombre de coronación del rey Amenofis III, "Nebmaatre", probablemente con la intención de remarcar quién era el propietario del palacio, y un posible signo de su deseo de propaganda política, en el sentido de intentar remarcar el papel de este rey como monarca poderoso capaz de llevar a cabo grandes obras y como protector de Egipto.

En el palacio de Malkata se documentan también motivos decorativos en los marcos de las puertas, en el remate de los muros, que se adornan con motivos florales, plantas, etc. ${ }^{10}$ Llama la atención que la decoración llega hasta los detalles más peque-

\footnotetext{
8 SCHÄFFER, Henry, Principles of Egyptian Art, Oxford, Oxford University Press, 1974, p. 1, fig. 3.

9 Sobre esta convención egipcia de representación en función de la posición del espectador, véase: ibid., p. 224.

10 SMITH, William Stevenson, Arte y arquitectura del Antiguo Egipto, Madrid, Oxford University Press, 2000 , pp. 263-265, figs. 280 (3) y 280 (4).
} 
ños del edificio, incluyendo dinteles de las puertas, balcones o ventanas, y no queda limitada a los grandes espacios, como los lienzos de los muros de las grandes salas. Si bien la decoración que cubre todo es habitual en templos y tumbas, sorprende que se decoren todos los elementos del palacio, incluso aquellos que no tienen una función de representación, sino un uso cotidiano, como las zonas de almacenaje. La decoración del palacio es profusa, lo que posiblemente da más libertad a los artistas, que pueden ensayar nuevos motivos. Además de la variedad de motivos iconográficos que encontramos, destaca que en cada sala aparece un nuevo diseño en el techo, sin que se repita el esquema compositivo. A pesar de la originalidad, los motivos de espirales y de rosetas tienen un lugar destacado, tal y como se deduce del análisis de los cientos de fragmentos llevado a cabo por la misión japonesa de Waseda. Como ejemplo mencionaremos la reconstrucción del esquema decorativo del techo de la sala N5 (fig. 3), en el que no parece casual que para las rosetas se hayan elegido los colores rojo y azul, tan típicos de frescos del Egeo.

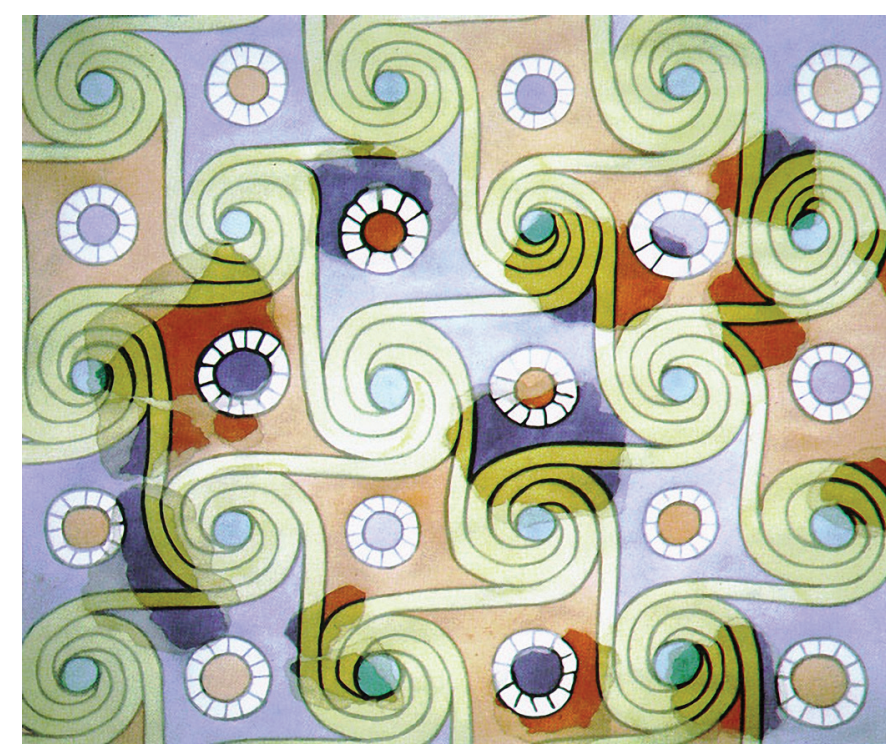

Fig. 3. Reconstrucción ideal del techo de sala N5, Palacio de Malkata, Reinado de Amenofis III, http://www.egyptpro.sci.waseda.ac.jp/e-mp.html.

Otra parte destacada del palacio con variadas pinturas es la zona de salas y dormitorios de las mujeres, que se ha venido llamando la "zona del harem", ya que comprende numerosas estancias para personajes de relevancia y para sus asistentes personales. Tanto la llamada sala con columnas del harem como una pequeña sala de audiencias junto a aquella, presentan sus suelos decorados con una composición basada en un rectángulo en el que se representaba un estanque en torno al cual se disponía un amplio borde en el que crecían plantas y las aves 
acuáticas volaban alrededor ${ }^{11}$. Este tipo de representaciones de estanques con una perspectiva aérea mostrando un paisaje acuático tiene una larga tradición en el arte egipcio ${ }^{12}$. También se atestigua pintura mural decorada en los aposentos de las mujeres, en una zona en la cual había almacenes con pequeños muros a modo de soportes para estantes, muros que se decoraban con escenas de novillos que saltan entre plantas de papiro, las cuales de nuevo son una muestra de este tipo de pintura naturalista ${ }^{13}$. Estas escenas no son muy habituales en la iconografía egipcia, siendo además estos temas de toros de nuestro interés por su vinculación con las pinturas murales de Tell el Dab'a (Avaris) en un palacio de comienzos de la XVIII dinastía ${ }^{14}$.

Lamentablemente apenas hay restos de las escenas que decoraban la parte superior de los muros del palacio, y que podrían revelar un programa iconográfico más complejo. Sólo se ha conservado una gran figura femenina en el muro occidental del segundo salón para audiencias, que se ha interpretado como una cortesana, que porta un elaborado tocado de flores. Se conserva además en esta sala un friso floral que recorre la parte superior del muro. Aunque no se conserva el resto de pinturas de la sala, tenemos una descripción de la misma, hecha por Daressy, quien descubrió los restos de pinturas de Malkata en $1888^{15}$. Este autor describía las pinturas diciendo que se representaba un toro corriendo por montañas, indicadas por bandas ondulantes azules, amarillas y rojas, salpicadas de rosetones rojos sobre el fondo azul y rosetones azules sobre el fondo rojo. El cielo raso estaba pintado con buitres en vuelo, con una composición similar a las de otras salas, como el primer salón para audiencias o el salón del harén ${ }^{16}$.

Resulta impactante la descripción que hizo Daressy de esa pintura mural de toros corriendo, ya que parece evocadora de uno de los temas más sorprendentes de las pinturas de Tell el Dab'a (Avaris). Como ya hemos señalado, se han encontrado en Avaris, (capital de los hyksos durante el II Período Intermedio, en el Delta Oriental del Nilo), un conjunto de pinturas murales con la técnica del fresco. La conexión de estas pinturas con el Egeo se basa en la técnica con la que fueron realizadas, pero sobre todo se basa en la temática. Los frescos, que decoraban los muros de un palacio de adobe de comienzos de la XVIII dinastía, presentan una temática muy significativa, como los motivos de rosetas o la imitación de mármol, en la que destaca sobre todo la temática de toros y hombres realizando saltos de toro que estaba en el palacio de Avaris (fig. 4). El motivo de la taurocatapsia tiene una larga tradición en el mundo egeo, siendo el máximo exponente el fresco de Knossos. Se ha señalado la existencia de un grupo de artistas minoicos en

11 NISHIMITO, Shin-ichi, «The Ceiling Paintings of the Harem Rooms at the Palace of Malqata», en Goettinger Miszellen, ${ }^{\circ} 127,1992$, pp. 69-80

12 SCHÄFFER, Henry (1974), op. cit., pp. 247-250.

13 SMITH, William Stevenson (2000), op. cit., pp. 263-264.

14 BIETAK, Manfred, MARINATOS, Nanno y PALYVOY, Clairy, Taureador Scenes in Tell el Dab'a (Avaris) and Knossos, Viena, Austrian Academy of Sciences, 2007.

15 DARESSY, Georges, "Le palais d'Aménophis III et le Birket Habou", en Annales du Service des Antiquités de l'Égypte, nº4, 1903, pp. 165-170.

16 SMITH, William Stevenson (2000), op. cit., p. 267. 


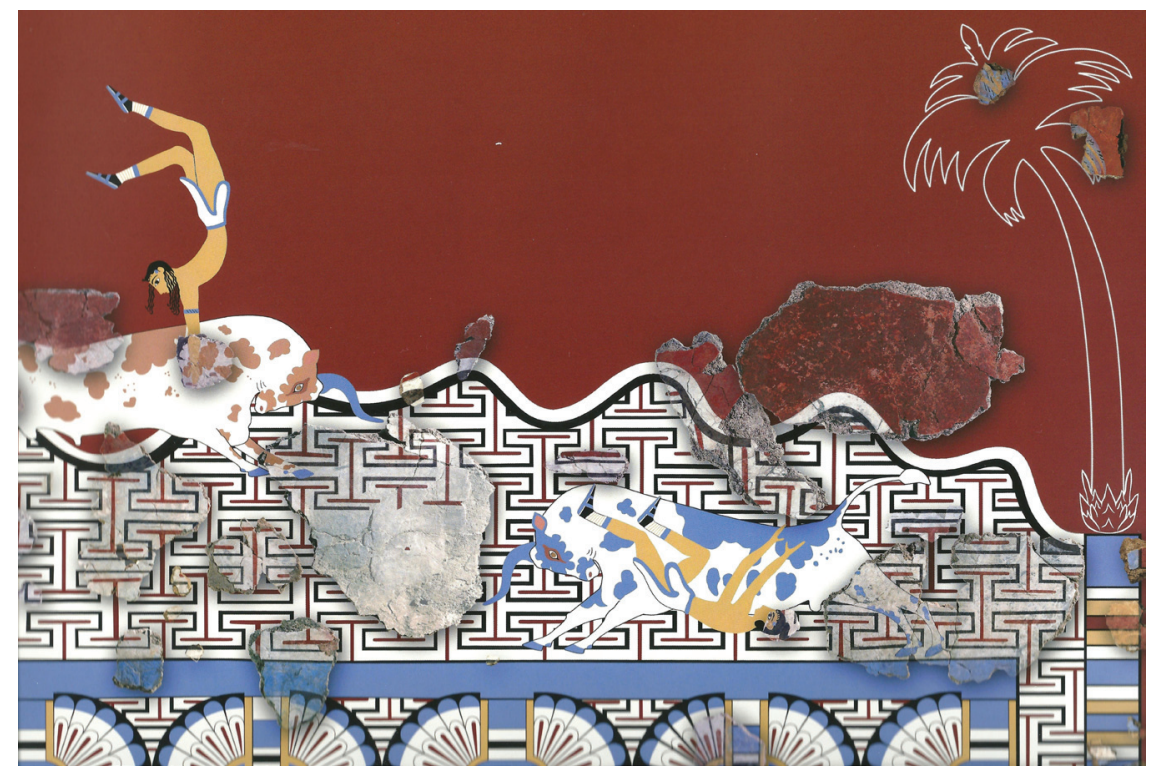

Fig. 4. Reconstrucción del panel con toros y laberinto de Avaris (Tell el Dab'a, Egipto), Inicios de la XVIII dinastía [tomado de BIETAK, Manfred, MARINATOS, Nanno, y PALYVOU, Clairy, Taureador Scenes in Tell el Dab'a (Avaris), Viena, Austrian Academy of Sciences Press, 2007, p. 59, fig. 59b].

Egipto, que habrían realizado los frescos en Avaris, cuya presencia en un palacio real egipcio sería, cuando menos, llamativa ${ }^{17}$.

Al analizar estas escenas de Malkata de novillos saltando entre plantas destaca el parecido con las representaciones de toros de Avaris, sobre todo por algunos ejemplos en los que se ve la postura de galope tendido tan típica del Egeo. La diferencia está en que en Malkata faltan las figuras humanas que realizan los saltos de toro, y da la sensación de que se ha olvidado el simbolismo de la taurocatapsia o que estas representaciones resultaban incomprensibles para el artista egipcio. Quizás los motivos de Avaris sirvieron de inspiración, y se copiaron algunos elementos pero ya sin comprender el significado original de las representaciones.

Estas composiciones con toros que vemos en Malkata recuerdan a las escenas de Avaris, y ello podría indicar que las de Avaris no son tan únicas y excepcionales como se creía, ya que de algún modo los artistas egipcios habrían asimilado algunos temas, y así se habrían conservado y perdurado algunos motivos iconográficos. No sólo los motivos de toros indicarían reminiscencias de los temas de taurocatapsia minoi-

17 BIETAK, Manfred, "Der Friedhof in einem Palastgarten aus der Zeit des späten mittleren Reiches und andere Forschungsergebnisse aus dem Östlichen Nildelta (Tell el Dab'a 1984-1987)", en Ägypten und Levante, II, 1991, pp. 47-75; BIETAK, Manfred, "Connections between Egypt and the Minoan World, New Results from Tell el Dab'a/Avaris”, en DAVIES, Vivian y SCHOFIELD, Louise (eds.), Egypt, the Aegean and the Levant, Londres, British Museum Press, 1995, pp. 19-23. 
ca, sino que también los rosetones podrían ser las típicas rosetas minoicas (también presentes en Avaris $)^{18}$, que se atestiguan además en otras pinturas conservadas en Malkata. Aunque sabemos que las pinturas de Avaris no se conservaron sobre los muros del palacio durante mucho tiempo, es lógico pensar que debieron de tener un fuerte impacto sobre quienes las contemplaron, y no sería raro pensar que artistas egipcios al servicio de la realeza pudieran haberlas visto.

Sin embargo, hay una diferencia básica entre las pinturas de Malkata y las de Avaris, y es que en Malkata hay diversos elementos típicamente egipcios, como las representaciones de Bes o los símbolos amuletos para la vida como el Ankj y el Sa. Encontramos en las pinturas de Malkata otros ejemplos de la temática tradicional egipcia, como las imágenes de cautivos, los buitres de alas extendidas, la representación de estanques, o la imagen del rey entronizado.

Respecto al estilo de las pinturas del palacio de Malkata, destacaremos algunos rasgos que comparte con las pinturas de Amarna. En concreto se atestiguan dos estilos de pincelada muy distinta. Por un lado, hay una pincelada libre en la que los motivos se tratan de manera muy superficial, y que se usa para la decoración de los bordes, o para los motivos de plantas y animales. Frente a este tipo de pintura más libre, sin restricciones, detectamos un diseño cuidadoso y detallado, que decora por ejemplo el friso de las palomas de la sala de audiencias de Malkata. De hecho, tanto en Malkata como en Amarna el mismo grupo de pintores parece haber utilizado ambos métodos de pincelada para tratar las representaciones naturalistas de plantas y animales que decoraban suelos, cielos rasos y espacios murales menores. De acuerdo con W. S. Smith, Akhenaton se llevaría a algunos de estos pintores que decoraron el palacio de Malkata a trabajar en Amarna ${ }^{19}$. Hay ciertos elementos de las pinturas de Amarna que parecen tener cierta relación con motivos egeos, lo que indicaría la pervivencia de la influencia egea. Podríamos decir que la llamativa decoración del palacio de Amarna se comprende en profundidad rastreando los antecedentes de Malkata, (si bien el arte amarniense es un fenómeno muy complejo con un importante contenido religioso y político).

Queremos prestar también atención a otro edificio que no forma parte del palacio de Malkata en sí, pero que está dentro del yacimiento y vinculado estrechamente a la figura de Amenofis III. Se trata de los restos de la llamada "estructura del primer jubileo" del monarca Amenofis III, construida con una finalidad muy particular, vinculada a la propaganda política del rey, y que fue demolida poco después de su erección debido a la ampliación del puerto de Birket Habu de Malkata ${ }^{20}$. Los restos de esta estructura real se hallaron en montones de deshechos bajo grandes montañas de tierra y cascotes producto de la excavación para ampliar el puerto, y que se habían

18 ASLANIDOU, Katherina, "Some Ornamental Scenes on the Wall Paintings from Tell el Dab'a: Iconography and Context”, en BIETAK, Manfred y CZERNY, Ernst (eds.), The Synchronisation of Civilisations in the Eastern Mediterranean in the Second Millennium B.C. III, Viena, Österreichische Akademie der Wissenschaften, 2007, pp.191-206.

19 SMITH, William Stevenson (2000), op. cit., p. 267.

20 JOHHSON, Raymond, "Monuments and Monumental Art under Amenhotep III: Evolution and Meaning”, en O'CONNOR, David y CLINE, Eric (eds.), Amenhotep III: Perspectives on his Reign, Ann Arbor, The University of Michigan Press, 1998, pp.75-76. 
dispuesto en filas ordenadas a lo largo del perímetro del puerto. Hay que destacar los numerosos restos de pinturas encontrados en el denominado yacimiento o zona $\mathrm{K}$, donde las excavaciones de 1973 sacaron a la luz cientos de fragmentos de enlucido de barro con decoración, que habían sido depositados en un contexto secundario, en el citado yacimiento $\mathrm{K}$ en los bordes del área palacial ${ }^{21}$.

El estudio de estos restos de pinturas del complejo del primer jubileo ha demostrado que la mayoría de los elementos iconográficos son egipcios en términos de técnica, estilo y temática. Sin embargo, un número considerable de elementos parece no corresponder a la tradición artística egipcia, sino que más bien tienen paralelos en motivos minoicos y micénicos, tales como la imitación de mármol, los paisajes rocosos, y el terreno relleno de rosetas ${ }^{22}$. Estas pinturas de nuevo sirven de punto de referencia para los motivos que aparecen en las pinturas de Tell el Dab'a, ya que existe cierta analogía entre ambas. De los fragmentos que la autora Margarita NicolakakiKentrou ha analizado, la mayoría proceden de un techo, y presentan en su mayor parte temas geométricos, que podrían tener relación con los motivos de los textiles del Egeo. Estos textiles eran muy apreciados en la época, tal y como demuestran las representaciones de keftiu, habitantes del Egeo representados en tumbas tebanas y que portan textiles junto con objetos de metales preciosos ${ }^{23}$. Es difícil valorar el papel de los textiles como medio de transmisión iconográfica entre Egipto y el Egeo de las composiciones geométricas presentes en Malkata, ya que los restos textiles en ambos ámbitos son muy escasos ${ }^{24}$.

Las pinturas de Malkata de la zona K también contienen temas que reflejan la iconografía de la realeza egipcia, como la representación de ciertos animales, escenas de caza, etc. En las pinturas de Avaris se han encontrado también escenas de caza, así como representaciones de felinos (animales vinculados a la realeza). Resulta muy significativo que estas pinturas de Malkata, que son un buen paralelo cercano a las de Avaris, tengan motivos y temática que se repiten, y que nos remiten de nuevo al Egeo.

En las pinturas de Malkata del área K encontramos gran similitud con los motivos de los frescos del Egeo en cuanto al modo de representar la naturaleza. Por ejemplo, se representa un relieve montañoso mediante una superficie ondulante, un motivo que aparece en Thera y en Tell el Dab'a. En esta representación de un terreno rocoso se utilizan los colores rojo, azul y amarillo, la misma combinación que se utiliza en el arte del Egeo. También encontramos la imitación de mármol, con la misma selección de colores que en el Egeo, el amarillo y el azul. La imitación de mármol se da asimismo en las pinturas del palacio de Mari, y en la decoración de un suelo en Alalakh, lo que sugeriría que este motivo decorativo se difundió en diferentes ámbitos. Respecto

21 NIKOLAKAKI-KENTROU, Margarita, "Malkata, Site K: The Aegean-related Motifs in the Painted Decoration of a Demolished Building of Amenhotep III", en HAWAS, Zahi y MILWARRD JONES, Angela (eds.), Eighth International Congress of Egyptologists, Abstracts of Papers, Cairo, International Association of Egyptologist, 2000, pp. 133-134.

22 Ibid., p. 133.

23 WACHSMANN, Shelley, Aegeans in the Theban Tombs, Lovaina, Peeters Publishers, 1987.

24 NICOLAKAKI-KENTROU, Margarita, "Affinities between the Aegeanizing Mural Motifs from Malkata's Site K and Contemporary Textile Icononography", Orientalia Lovaniensia Analecta, ${ }^{\circ} 150,2007$, pp. 1381-1390. 
al mencionado terreno que aparece rellenado por rosetas, debemos decir que sirve de fondo a la figura de un león con una gran melena, representado a la manera del Egeo. Lo excepcional no es la presencia de la imagen del león, animal con gran tradición en la iconografía egipcia, sino el estilo de representarlo y sobre todo que las rosetas sirven de fondo, algo que no se da en el arte egipcio. En cambio, en el arte del Egeo sí se combinan frecuentemente los motivos de animales y de rosetas de fondo.

Margarita Nicolakaki-Kentrou ha intentado realizar una reconstrucción veraz de cómo serían las composiciones de Malkata con escenas de toros y fondo de rosetas (fig. 5), que recuerda mucho a las composiciones de Avaris (fig. 4). Si comparamos las pinturas de un lugar y otro, vemos que no son copias, sino que comparten cierto estilo y sobre todo la presencia de toros como motivo decorativo principal.

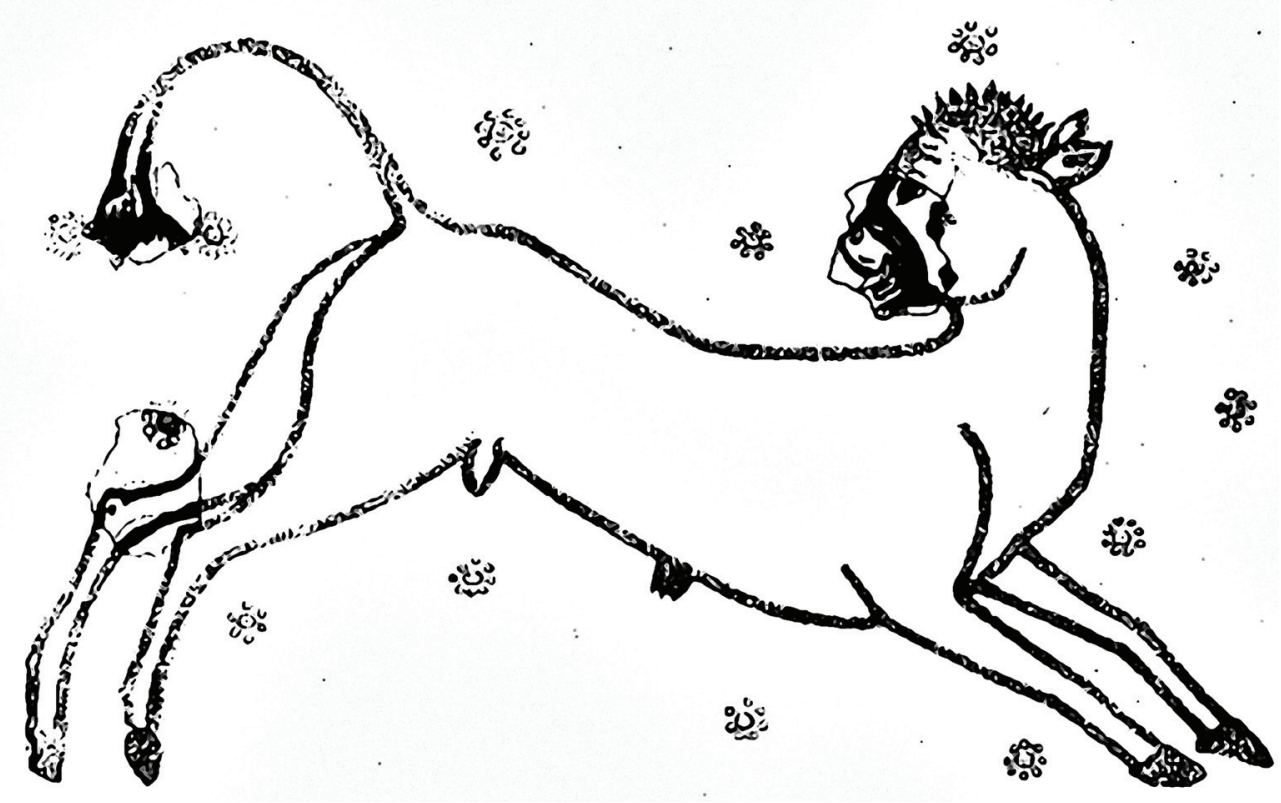

Fig. 5. Reconstrucción ideal del panel con novillo y rosetas, Palacio de Malkata, Reinado de Amenofis III (1391-1393 a.C.) [tomado de NIKOLAKAKI-KENTROU, Margarita, "Malkata, Site K: The Aegean-related Motifs in the Painted Decoration of a Demolished Building of Amenhotep III”, en HAWAS, Zahi y MILWARD JONES, Angela (eds.), Eighth International Congress of Egyptologists, Abstracts of Papers, Cairo, International Association of Egyptologist, 2000, pp. 133-134].

Tras el análisis de los motivos iconográficos más destacados de las pinturas de Malkata y sus paralelos, es posible esbozar algunas conclusiones, por ejemplo respecto al programa iconográfico de las distintas áreas palaciales. Da la sensación de que la temática iconográfica de las salas con función pública, tales como las salas de audiencias, tienen más conexión con la tradición egipcia, y en las zonas no representativas 
se introducen otras temáticas, con elementos y modos de representación animal que nos remiten al Egeo. Quizás en esas zonas el artista gozaba de más libertad y se podía tomar ciertas licencias. Posiblemente en las zonas de ámbito privado el repertorio iconográfico no debía ceñirse a los temas fijos y habituales de la pintura egipcia, es decir, no había un estereotipo de representaciones fijas. Este tipo de innovaciones probablemente no se dan por casualidad en un edificio palacial, es algo que posiblemente no tenía cabida en las pinturas murales de las tumbas. Son numerosos los ejemplos de pinturas murales del Reino Nuevo del ámbito funerario, que además han llegado a nuestros días en un estado de conservación bueno, ya que suelen ser realizadas en tumbas excavadas en la roca. En estas pinturas murales funerarias las representaciones estaban ligadas al difunto, a las creencias religiosas de vida en el más allá, o a las acciones importantes que el difunto había llevado a cabo en vida, resultando todo ello en un repertorio iconográfico más reducido, no tanto por las limitaciones artísticas del pintor, sino por la elección de temas apropiados. Ello no implica que el arte del ámbito funerario permaneciese inalterable, ya que se atestiguan novedades de estilo y de iconografía desde la época tutmósida.

También queremos apuntar algunas ideas sobre la existencia motivos de estilo egeo en las pinturas de Malkata, pues lógicamente surgen varios interrogantes, no sólo respecto al origen de los artistas que realizaron estas pinturas sino también respecto a los mecanismos de transmisión artística ${ }^{25}$. Por ejemplo la combinación de colores (rojo, azul y amarillo) que aparece en las pinturas de Malkata se da sólo en los frescos del Egeo, lo que sugiere un contacto entre Egipto y algunos de los palacios minoicos. Dada la existencia de las pinturas con estilo y técnica del Egeo halladas en Avaris de época muy próxima, nos preguntamos si los artistas egipcios habituales al servicio de la monarquía egipcia pudieron haber contemplado los frescos que decoraban el palacio. Sabemos que las pinturas de Avaris no duraron mucho decorando las paredes del edificio palacial: se cree que después de unas décadas las pinturas se desprendieron por sí solas ${ }^{26}$, posiblemente porque se hicieron sobre paredes de adobe que no eran el soporte habitual de los frescos ni el más idóneo. Si tenemos en cuenta que los pintores egipcios solían ser artesanos especializados y escasos a quienes se encomendaba la decoración de una tumba o una zona palacial, no debe parecer descabellada la idea de que una escuela de pintores egipcios hubiese contemplado las pinturas de Tell el Dab'a.

Pero por otro lado ello no implica que se deba descartar un contacto directo entre los dos ámbitos, es decir, que hubiese un contacto directo entre la corte de Amenofis III y el ámbito egeo. Puede que este contacto entre Egipto y el Egeo sea lo que reflejan los topónimos egeos que aparecen en el complejo de Kom el Khetan de Amenofis $\mathrm{III}^{27}$, interpretados como la evidencia de una embajada egipcia realizando un recorri-

25 NIKOLAKAKI-KENTROU, Margarita (2000), op. cit., pp. 133-134.

26 BIETAK, Manfred, "Tell el Dab'a and the Aegean Tell el Dab'a and the Aegean World", en Annual of the British School of Athens, 2000, p. 194.

27 Sobre el tema de la lista de Kom el Khetan, véase por ejemplo: EDEL, Elmar, Die Ortsnamenlisten aus den Totentempel Amenophis III, Bonner Biblishe Beiträge, Band 85, Leiden, 1966; JAMES, Thomas Garnet Henry, "Aegean Place-Names in the Mortuary Temple of Amenofis III at Thebes", en Bulletin of the Institute of Classical Studies, 18, 1970, pp. 144-145; ÖSING, Jurgen, "La liste des toponymes Égeens au Temple 
do por el Egeo, (idea apoyada en los objetos de Amenofis III hallados en el Egeo ${ }^{28}$ ). Es posible incluso que durante el Reino Nuevo se produjese un viaje de artistas egipcios al Egeo, que traerían consigo nuevas ideas para los repertorios decorativos.

Debemos señalar que el período de Amenofis III destaca por un auge de las artes, debido en parte a la gran actividad constructiva, que tiene pocos paralelos en los reinados anteriores. Sorprende la gran escala de los edificios de esta época, lo que parece ser una innovación del reinado de Amenofis III. En relación con el arte pictórico, las artes decorativas y las artes menores alcanzan un gran desarrollo, tanto en calidad como en cantidad. Las innovaciones de estilo que hemos visto en el palacio de Malkata no implican, sin embargo, que los estilos más tradicionales desaparezcan o dejen de utilizarse (un buen ejemplo de este arte más tradicional lo tenemos en la tumba de Ramose, TT 55). Parece que existe una convivencia de estilos y de tendencias artísticas, que es prueba de un período de actividad y de pluralidad ${ }^{29}$. Respecto al arte de su reinado, y en relación al distintivo y revolucionario arte de la época de Amarna, John Baines ha señalado que muchos de los desarrollos e innovaciones del tiempo de Amenofis III tenían sus raíces en siglos anteriores. Sin embargo, en la posterior época amarniense parece darse un punto culminante tanto en lo religioso como en lo artístico, sería la culminación de una tendencia a la cual contribuyen factores como un largo período de paz, una riqueza en aumento y amplios contactos entre la élite. El resultado de esa tendencia es un "estilo internacional" de un tipo que no se había atestiguado antes ${ }^{30}$.

Quizás el hallazgo de restos de pinturas minoicas en Tell el Dab'a, y de ciertos motivos o estilo minoico en Malkata podrían ser consecuencia de la llegada de artistas minoicos o de la influencia minoica a comienzos de la XVIII dinastía. El palacio de Avaris fue inicialmente vinculado al reinado de Ahmose, (1550-1525 a.C) $)^{31}$, aunque la hipótesis más reciente de los investigadores de Tell el Dab'a fecha el palacio en época tutmósida ${ }^{32}$. Esta nueva fecha tutmósida ubica las pinturas de Avaris es un período de constatado contacto con el Egeo, como demuestran las representaciones de keftiu en las tumbas tebanas. Si las pinturas de Avaris son del período tutmósida, el lapso de tiempo hasta el reinado de Amenofis III es más corto aún, y la influencia sería

Funeraire d'Amenophis III", en ÖSING, Jurgen (ed.), Aspects de la Culture Pharaonique. Quatre leçons au College de France, París, Diffusion de Boccard, 1992, pp. 25-36; CLINE, Eric H. y STANNISH, Steven M., "Sailing the Great Green Sea? Amenhotep III's Aegean List from Kom el-Hetan, Once More", en Journal of Ancient Egyptian Interconnections, Vol, 3:2, 2011, pp. 6-16.

28 PHILLIPS, Jack, y CLINE, Eric, “Amenhotep III and Mycenae: New Evidence”, en DAKOURIHILD, Anastasia y SHERRAT, Susan (eds.), Autochthon, Papers presented to O.T.P.K. Dickinson, Oxford, Archaeopress, 2005, pp. 317-328; KELDER, Jorrit, "The Egyptian interest in Mycenaean Greece", en Jaarbericht Ex Oriente Lux, 2010, pp. 125-140.

29 BAINES, John, “The Dawn of the Amarna Age", en O'CONNOR, David y CLINE, Eric (eds.), Amenhotep III: Perspectives on his Reign, Ann Arbor, The University of Michigan Press, 1998, p. 305.

30 Ibid., pp. 310-311.

31 BIETAK, Manfred y MARINATOS, Nanno, "The Minoan Wall Paintings from Avaris", en Ägypten und Levante, V, 1995, pp. 49-62.

32 BIETAK, Manfred, "Minoan Artists at the Court of Avaris (Tell el Dab'a)", en ARUZ, Joan, BENZEL, Keith y EVANS, John, (eds.), Beyond Babylon. Art, Trade and Diplomacy in the Second Millennium B.C., New York / New Haven, Metropolitan Museum of Art / Yale University Press, 2008, pp. 249-250. 
mucho más directa. Si bien parece que los artistas de Avaris procedían del Egeo, en cambio es muy probable que los que realizaron las de Malkata o las de Amarna fueran egipcios, como sugiere la técnica utilizada. Es posible que las técnicas importadas del Egeo se perdiesen, pero que los motivos corrieran mejor suerte y sobrevivieran, tal vez también gracias a la costumbre de utilizar los llamados "libros de modelos" donde los artistas registraban los motivos iconográficos, libros que posiblemente pasaban de una generación a otra. En cualquier caso, las pinturas de Malkata tienen un cierto "sabor egeo" que nos sirve de punto de comparación para pinturas halladas en el palacio de Tell Kabri en Siria, y para las de Tell el Dab'a, y en definitiva para comprender el fenómeno del intercambio de artistas entre el Egeo, Egipto y el Próximo Oriente ${ }^{33}$.

Hace ya varias décadas destacados investigadores como Helene Kantor o Stevenson Smith propusieron que la influencia egea en las pinturas de Malkata (y también de Amarna) podía proceder de contactos anteriores con el Egeo, una hipótesis que toma fuerza a la luz de los nuevos hallazgos ${ }^{34}$. Como Lyvia Morgan ha destacado, las pinturas de Avaris pueden considerarse el primer y crucial ejemplo de los vínculos que nos faltaban ${ }^{35}$. Debemos tener en cuenta que las influencias artísticas e iconográficas entre Egipto y el Egeo son un proceso complejo pero con larga trayectoria que se remonta al menos al Reino Medio ${ }^{36}$. Gracias a nuevos hallazgos como los de Avaris, podemos analizar las pinturas de Malkata desde una nueva perspectiva y contemplarlas con nuevos ojos, en un renovado intento de reinterpretar el arte egipcio.

33 NIEMEIER, Wolfgang, NIEMEIER, Barbara, "Minoan Frescoes in the Eastern Mediterranean", Aegaeum, 18, 1998, pp. 69-97, especialmente p. 96.

34 KANTOR, Helene, "The Aegean and the Orient in the Second Millenium B.C.", en American Journal of Archaeology, 51, 1947, 83-84.; SMITH, William Stevenson, Interconnections in the Ancient Near East, New Haven- Londres, Yale University Press 1965, pp. 161-162.

35 MORGAN, Lyvia, "Minoan Painting and Egypt. The Case of Tell el Dab'a", DAVIES, Vivian , SCHOFIELD, Louise, (eds.), Egypt, the Aegean and the Levant, Londres, British Museum Press, 1995, pp. 29-53.

36 Posiblemente esta influencia se inicia ya en el Reino Medio, con la difusión de pequeños objetos que tienen una imagen peculiar, como por ejemplo los escarabeos cuya forma resulta atractiva para los minoicos, o ciertas combinaciones de motivos egeos que vemos en los techos de tumbas del Reino Medio, inspirados en textiles. 